\title{
Impact of empagliflozin on subclinical left ventricular dysfunctions and on the mechanisms involved in myocardial disease progression in type 2 diabetes: rationale and design of the EMPA-HEART trial
}

\author{
Andrea Natali ${ }^{1}$, Lorenzo Nesti ${ }^{*} \mathbb{0}$, lacopo Fabiani ${ }^{2}$, Enrico Calogero ${ }^{2}$ and Vitantonio Di Bello ${ }^{2}$
}

\begin{abstract}
Background: Asymptomatic left ventricular (LV) dysfunction is highly prevalent in type 2 diabetes patients. Unlike the other hypoglycemic drugs, SGLT2 inhibitors have shown potential benefits for reducing cardiovascular death and risk factors, aside from lowering plasma glucose levels. With this study we aim at determining whether the treatment with empagliflozin is associated with an improvement in LV functions in diabetic patients with asymptomatic LV dysfunction against Sitagliptin, which is presumably neutral on myocardial function. To determine changes in LV systolic and diastolic functions we will use speckle-tracking echocardiography, a novel sensitive, non-invasive, bedside method allowing the calculation of LV global longitudinal strain (GLS), an index of myocardial deformability, as well as 3D echocardiography, which allows a better evaluation of LV volumes and mass.
\end{abstract}

Methods: The EMPA-HEART trial will be a phase III, open label, active-controlled, parallel groups, single centre, exploratory study conducted in Pisa, Italy. A cohort of 75 diabetic patients with normal LV systolic (2D-Echo EF > 50\%) and renal (eGFR sec MDRD > $60 \mathrm{ml} / \mathrm{min} / 1.73 \mathrm{mq}$ ) functions and no evidence of valvular and/or ischemic heart disease will be randomized to either Empagliflozin $10 \mathrm{mg} /$ die or Sitagliptin $100 \mathrm{mg} /$ die. The primary outcome is to detect a change in GLS from baseline to 1 and 6 months after treatment initiation. The secondary outcomes include changes from baseline to 6 months in 3-D Echocardiography EF, left atrial volume and $E / E^{\prime}, V_{2}$ max as measured at cardiopulmonary test, cardiac autonomic function tests ( $R-R$ interval during Valsalva manoeuvre, deep-breathing, lying-tostanding), and the determination of a set of plasma biomarkers aimed at studying volume, inflammation, oxidative stress, matrix remodelling, myocyte strain and injury.

Discussion: SGLT2 inhibitors might affect myocardial functions through mechanisms acting both directly and indirectly on the myocardium. The set of instrumental and biohumoral tests of our study might actually detect the presence and entity of empagliflozin beneficial effects on the myocardium and shed light on the mechanisms involved. Further, this study might eventually provide information to design a clinical strategy, based on echocardiography and/ or biomarkers, to select the patients who might benefit more from this intervention.

Trial registration EUDRACT Code 2016-0022250-10

Keywords: Empagliflozin, SGLT2, Mycardial dysfunction, GLS, Speckle-tracking, Cardiovascular

\footnotetext{
${ }^{*}$ Correspondence: Iorenzonesti90@gmail.com

1 Department of Clinical and Experimental Medicine, Pisa University, Via

Savi 27, 56100 Pisa, Italy

Full list of author information is available at the end of the article
} 


\section{Background}

\section{Diabetes mellitus and myocardial dysfunction}

The pathogenesis of myocardial dysfunction in type 2 diabetes mellitus (T2D) is multifactorial, being the diabetic myocardium chronically exposed to a mixture of metabolic, haemodynamic, macro and microvascular insults, which altogether are responsible for the greater incidence of heart failure (HF) and cardiovascular death of these patients [1]. The peculiar complex and multifactorial pathogenesis of HF in T2D patients fit into a disease-specific condition named diabetic cardiomyopathy, which may be found in isolation or associated to coronary artery and/or hypertensive heart disease $[2,3]$. In this scenario, is not surprising that asymptomatic left ventricular (LV) dysfunction, defined as either systolic or diastolic abnormalities in the absence of clinically detectable cardiac disease, is highly prevalent in T2D patients, with estimates ranging from 50 to $70 \%[4,5]$. A better knowledge of its early-stage risk factors and its natural evolution would allow the design of effective prevention strategies as strongly recommended by current clinical guidelines, which emphasize the importance of early diagnosis and interventions in subjects at risk [3]. However, one major limitation to this approach is the lack of consensus with regard to the best method to detect asymptomatic LV dysfunction, which is responsible for the limited information, particularly about the effects of antidiabetic drugs on LV performance. The available methods for accurately measuring cardiac functions are either too complex (cardiac magnetic resonance) or not sensitive enough (brain-derived natriuretic peptideBNP-, 2D ejection fraction-EF- and tissue Doppler) to detect subtle changes in cardiac performances, particularly in asymptomatic subjects.

\section{Sodium-glucose linked co-transporter 2 (SGLT2) inhibitors in cardiovascular disease}

In the kidney, plasma glucose is freely filtered into the urine through the glomerulus and is reabsorbed in the tubule through an efficient system of ATP-dependent transporter proteins, the sodium-glucose-linked cotransporters (SGLT). SGLTs are found in the proximal tubule in two different isoforms, namely SGLT1 and SGLT2. Both proteins bind and transport sodium and glucose, SGLT2 in a one-to-one and SGLT1 in a two-toone ratio, while showing some clinically relevant differences. SGLT2, a high-capacity, low-affinity transporter, is present in segment 1 of the tubule and normally accounts for almost $90 \%$ of the entire kidney glucose reuptake. In T2D, secondary to chronic hyperglycemia, the expression of SGLT2 is upregulated, leading to an increased renal tubular reabsorption exacerbating hyperglycemia [6]. Conversely, SGLT1 is a low-capacity, high-affinity transporter present in segment 3 of proximal tubule, but that is also expressed, to a greater extent than in the kidney, in the small intestine and in the heart [7]. In the latter, SGLT1 is thought to be localized both in cardiomyocytes and in capillaries [8]. Besides their proven efficacy in lowering plasma glucose levels, SGLT2 inhibitors have also been shown to have potential benefits for improving other cardiovascular risk factors, such as body weight and blood pressure, while being well tolerated [9]. The study EMPA-REG, quite unexpectedly, reported in the diabetic population a $35 \%$ reduction in hospitalization for $\mathrm{HF}$ and a $32 \%$ reduction in cardiovascular death largely due (data from the Supplementary appendix of ref [10]) to a reduction in fatal events related either to worsening of HF (incidence rates: 0.2 vs $0.8 \%$ ) or to sudden death (incidence rates: $1.1 \mathrm{vs} 1.6 \%$, in the pooled empagliflozin and placebo group, respectively), while outcomes more closely related to atherosclerosis, e.g. cerebrovascular and ischemic heart diseases, were only marginally affected. In addition, the benefit was evident very early after treatment initiation ( $\sim 6$ months). Since other oral hypoglycemic drugs have been shown either to increase the risk of HF [11, 12], or to be neutral $[13,14]$, then the hypothesis that empagliflozin might directly and rapidly improve LV function, irrespective of the improvement in metabolic control, has been raised. Similar results on HF have been recently reported with another SGLT2 inhibitor, canagliflozin, in the recently published CANVAS study [15] and also in a large observational study on users of SGLT-2 inhibitors (CVD-REAL) in which the majority of the subjects were treated with dapagliflozin [16]. Diastolic function in ob/ob in a preliminary report in 10 patients of EMPA-HEART study short-term (151 days) with empagliflozin was associated with a $15 \%$ reduction in LV mass index and a $13 \%$ increase in early lateral annular tissue Doppler velocity $\left(\mathrm{e}^{\prime}\right)$, a proxy of LV compliance. Finally, the recent article by Januzzi et al. [17] demonstrated that chronic therapy with Canagliflozin is associated with delayed physiologic rise in serum NT-proBNP and high sensitive troponin I (hsTnI) in a 2 years'-follow up in older T2D patients by a little but significative amount, thus suggesting a beneficial role. Despite this, information with respect to SGLT2 inhibitors effect on myocardial function is very limited.

\section{Speckle-tracking and 3D echocardiography for early detection of myocardial dysfunction}

A recent sensitive, non-invasive and bedside method for the evaluation of myocardial systolic and diastolic function, namely 2D-speckle-tracking echocardiography (STE), has recently been developed and validated (against tissue Doppler, cardiac magnetic resonance and sonomicrometry) in large cohorts of patients, including subjects 
with T2D [18-21]. STE exploits the natural acoustic markers that are evident in grey-scale ultrasound images within the myocardial tissue, which corresponds to specific anatomical structures [22]. The spatial displacement of the speckles during the cardiac cycle reflects the strain of the myocardium and can be tracked on a frame-by-frame basis for each region of interest both in longitudinal and radial directions according the standard echocardiographic approaches. Strain values are calculated for each standard myocardial segment (segmental strain) and the average value of all segmental strains provides the global LV strain.

Currently only LV global longitudinal strain (GLS) has been validated. Interestingly, among T2D outpatients without valvular disease, previous cardiac events or symptoms, and with a normal 2D EF, an abnormal GLS measured by STE has been consistently found in $30-50 \%$ of the subjects [23-25]. Of note, in T2D patients the condition of normal EF and abnormal GLS was associated with a reduced $\mathrm{VO}_{2}$ max at cardiopulmonary test [20], and, most importantly, to an adverse cardiovascular outcome at 10-year follow-up [26].

Very recently, 3D echocardiography has also become available for clinical use. Combined matrix array transductors allow contemporary and real-time bedside evaluation of 2D and 3D images. Being more accurate with respect to $2 \mathrm{D}$ echography, which relies on a set of approximate geometric assumptions, it currently represents a promising tool in the measurement of both systolic and diastolic volumes, as well as LV mass and remodeling itself [27].

\section{Biomarkers of cardiac function and mechanisms of disease} In order to detect subclinical LV dysfunction and to provide information on the mechanisms potentially implicated in the diabetic cardiac dysfunctions, we have chosen a set of plasma biomarkers, which not only look at specific pathways (volume, inflammation, oxidative stress, matrix remodelling, myocyte strain and injury) but were also validated against hard endpoints [28].

\section{Volume biomarkers and total body water (TBW)}

We will measure plasma aldosterone (DiaSorin, Saluggia, Italy; intra-assay $9.7 \%$ and inter-assay $11.5 \%$, n.v. 3.5-30.0 ng/dl) and renin (CisBIO, Bedford, MA, USA; intra-assay $1.8 \%$ and interassay $4.0 \%$, n.v. $5.1-59.4 \mathrm{pg} /$ $\mathrm{ml}$ ) as a proxy for rennin-angiotensin-aldosterone system (RAAS) activation, which track effective circulating volume. The activation of the RAAS system also has been shown to be a prognostic marker in patients with HF. In a cohort of 996 subjects [29] plasma renin has been shown to predict death at 3 years follow-up, independently of the most important predictors: age, EF and
NT-pro-BNP. The body fluid status will be assessed also by measuring total body water (TBW) using the deuterated water dilution method [30]. A fixed amount (10 g) of 99\% deuterated water (Sigma Aldrich) will be ingested at 10:00 p.m. and the patient will collect a urine sample the subsequent morning at 7:00. TBW will be calculated as the deuterium dilution space divided by 1.04, to correct for exchange of the deuterium label with non-aqueous $\mathrm{H}$ of body chemicals [31]. Isotope abundances in urine were determined in duplicate by nuclear magnetic resonance $\left({ }^{2} \mathrm{H}\right.$ NMR $)$ according to the protocol described by Khaled et al. [32].

\section{Myocardial parietal stress biomarkers}

We will measure natriuretic peptides (NPs); namely brain natriuretic peptide (BNP) and N-terminal pro-brain natriuretic peptide (NT-proBNP) that are released into the bloodstream in direct proportion to the mechanical stress of the myocardium [33]. As such, NPs have been proved useful in screening asymptomatic subjects at risk of developing HF, such as the elderly and those with hypertension, diabetes, or asymptomatic coronary artery disease [33, 34], and will be used in this study for grading and monitoring the changes in asymptomatic LV dysfunctions observed with STE. More recently also proadrenomedullin has been validated as a myocardial parietal stress plasma biomarker. This cardiomyocytederived peptide is released in proportion to cardiac pressure and volume [35] and its serum levels are useful for grading HF severity and predict death, either alone or when combined with NT-proBNP [36].

\section{Cardiomyocyte injury biomarkers}

Plasma troponin- $\mathrm{T}$ will be measured to assess cardiomyocyte injury being present in HF patients not only as a result of ischemia, but also as a consequence of inflammation, oxidative stress, and neurohormonal activation. In fact, with the high-sensitive assay, abnormal troponin levels can be detected in up to $92 \%$ of HF patients, wherein are of high prognostic value being associated with an increased risk of death even after adjustment for baseline variables and BNP [37].

\section{Inflammation/oxidative stress biomarkers}

High-sensitivity C-reactive protein (hsCRP) is an acutephase reactant synthesized by hepatocytes in response to the proinflammatory cytokine interleukin-6 (IL-6) [38] in a variety of inflammatory processes. Its serum levels appear to be positively associated with HF severity and progression being an independent predictor of adverse outcomes in patients with acute or chronic HF [39, 40]. Further, notwithstanding its non-specificity, CRP might have a causal role in vascular and/or myocardial 
damage, since it exerts direct adverse effects on the vascular endothelium by reducing nitric-oxide release and increasing endothelin-1 production, as well as by inducing expression of endothelial adhesion molecules [41]. Also tumor necrosis factor-alpha (TNF- $\alpha$ ) has been proposed as a marker of HF and asymptomatic LV dysfunction. This pro-inflammatory cytokine has been observed to cause LV dilatation, seemingly by activating matrix metalloproteinases [42]. Despite the fact that the blockade of TNF- $\alpha$ did not result in clinical benefit in patients with HF [43], TNF- $\alpha$ levels have been then proposed to predict the future development of HF in asymptomatic elderly subjects [44]. Together with inflammation, also oxidative stress plays a role in determining myocardial damage. Since reactive oxygen species are many and are difficult to measure we focused on the plasma biomarkers that have been validated in clinical trials: myeloperoxidase (MPO). The levels of plasma MPO, in fact, are elevated in coronary artery disease, possibly having a role in determining and revealing LV remodeling [45]. Moreover, its levels correlate with the severity of $\mathrm{HF}$ and are independent predictors of death from HF, even after adjustment for baseline variables [46].

\section{Matrix remodelling biomarkers}

Being adverse myocardial remodelling largely dependent from excessive collagen synthesis and associated with impaired LV function and adverse clinical outcomes in patients with HF, we decided to measure type III pro-collagen, whose serum level has been shown to be an independent predictor of adverse outcomes in patients with HF [47].

\section{Methods}

\section{Rationale and aim of the study}

The specific medical need addressed by this study is to offer T2D patients, who appear to be at high risk for developing HF and its lethal consequences, a new therapeutic option capable not only of controlling their plasma glucose but also improving their underlying subclinical cardiomyopathy. Further, this study might eventually provide information to design a clinical strategy, based on echocardiography and/or biomarkers, to select the patients who might benefit more from this intervention.

With this study we aim at verifying whether, in T2D patients with subclinical cardiomyopathy (i.e. without: overt HF symptoms and signs, significant valvular heart disease, evidence of inducible myocardial ischemia and with 2 -D ejection fraction $\geq 50 \%$ ) the treatment with empagliflozin is associated with an improvement in LV systolic function, as measured by GLS through STE, in comparison to sitagliptin, an equally effective plasma glucose lowering agent, which has been shown to be neutral on HF-related events prevention.

\section{Study design}

The EMPA-HEART trial will be a phase III, open label, active-controlled, parallel groups, single centre, exploratory study conducted in Pisa, Italy (Fig. 1). This is a proofof-concept study aiming at evaluating whether the chronic treatment with the SGLT-2 inhibitor empagliflozin has an effect on myocardial function above and beyond its effect on metabolic control. The patients will be randomized to either empagliflozin $10 \mathrm{mg} / \mathrm{die}$ or to Sitagliptin $100 \mathrm{mg} /$ die as an active comparator, which has a similar potency in terms of glucose control and, on the bases of large clinical trials, does not influence the risk of developing HF. The study consists of 7 visits (see Table 1) and will last 2 years, therefore, being the start programmed for July 2017 the end of the study is expected for July 2019.

\section{Study population}

Volunteers will be recruited among patients attending visits at the Diabetes Outpatient Unit of Internal Medicine at the Pisa University Hospital (AOUP). The experimental sessions will take place in the Laboratory of Nutrition, Metabolism and Atherosclerosis and part in the unit of Cardio-angiology of the AOUP under the responsibility of AN and VD, respectively. All potential participants who meet the following criteria will be eligible for the trial:

\section{Inclusion criteria}

- Male or female affected by type 2 diabetes mellitus (T2D).

- Age $\geq 40$ and $\leq 80$ years.

- $\mathrm{HbA}_{1 \mathrm{c}}$ levels $\geq 53$ (7\%) and $\leq 69 \mathrm{mmol} / \mathrm{mol}$ (8.5\%).

- On stable (since 3 months) antidiabetic therapy with either Metformin alone or Metformin + basal insulin (this constraint is determined by the present Italian prescription rules).

- On stable (since 3 months) cardio-active therapies (e.g. anti-hypertensive drugs, diuretics, drugs for asthma or migraine prophylaxis).

- Preserved kidney function as defined by eGFR $\geq 45 \mathrm{ml} \mathrm{min}^{-1} 1.73 \mathrm{~m}^{2}$.

- Preserved left ventricular function as defined by $\mathrm{EF} \geq 50 \%$.

\section{Exclusion criteria}

- Refuse or inability to give informed consent.

- Patients unlikely to comply with the protocol or unable to understand the nature, scope and possible consequences of the study. 

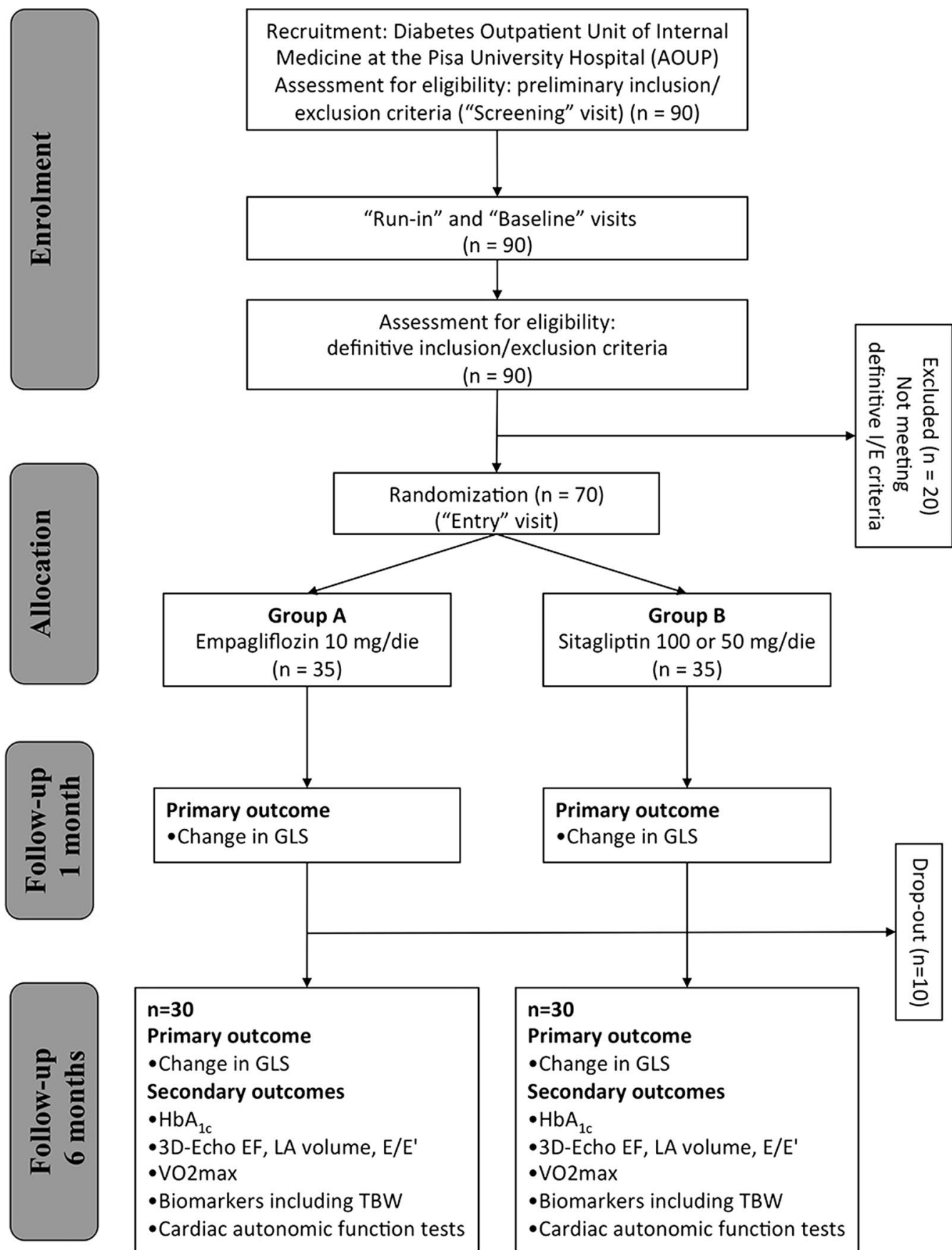

Fig. 1 Study design flowchart. EF ejection fraction, GLS global longitudinal strain, $H b A_{l c}$ glycated haemoglobin, LA left atrium, TBW total body water, $\mathrm{VO}_{2}$ max $\mathrm{O}_{2}$ consuption at peak of stress

- Employees of the investigator or study centre (i.e. principal investigator, sub-investigator, study coordinators, other study staff, employees, or contractors of each), with direct involvement in the proposed study or other studies under the direction of that investigator and/or study centre, as well as family members of the employees or the investigator.
- Patients with type 1 diabetes mellitus.

- Pregnancy or active breast feeding.

- History of hospitalization for acute coronary syndrome or heart failure.

- Respiratory insufficiency or history of clinically significant respiratory diseases (chronic obstructive pulmonary disease). 
Table 1 Study conduction flowchart overview of all visit and tests scheduled for participants of the EMPA-HEART trial

\begin{tabular}{|c|c|c|c|c|c|c|c|}
\hline Visits & Screening & Run-in & Baseline & Entry & 1 Month & 6 Months & Study end \\
\hline Time (week) & -5 & -3 & -1 & 0 & 4 & 24 & 26 \\
\hline Medical history & & x & & & & & $x$ \\
\hline Physical examination & & $x$ & & $x$ & $x$ & & $x$ \\
\hline ECG & & $x$ & & & & & $x$ \\
\hline Preliminary I/E criteria & $x$ & & & & & & \\
\hline Definitive I/E criteria & & & & $x$ & & & \\
\hline Informed consent & $x$ & & & & & & \\
\hline Randomization & & & & $x$ & & & \\
\hline Drug prescription & & & & $x$ & & & \\
\hline Biomarkers & & $x$ & & & & & $x$ \\
\hline Body water assessment & $x$ & & & & & & $x$ \\
\hline Safety sample & & $x$ & & & & & $x$ \\
\hline Drug compliance & & & & & & & $x$ \\
\hline Adverse effect & & & & & $x$ & & $x$ \\
\hline Cardiac autonomic tests & & $x$ & & & & & $x$ \\
\hline Exercise test & & & $x$ & & & $x$ & \\
\hline Echocardiography & & & $x$ & & $x$ & $x$ & \\
\hline
\end{tabular}

- NYHA class III and IV or other symptoms of heart failure (breathlessness, orthopnoea, paroxysmal nocturnal dyspnoea, fatigue, increased time to recover after exercise, ankle swelling) unrelated to most common non-cardiac causes.

Signs of heart failure (elevated jugular venous pressure, third heart sound, laterally displaced apical impulse).

- Acute or chronic inflammatory diseases.

- History of active neoplastic disease within the last 5 years.

- Patients with known hypersensitivity to empagliflozin and its excipients.

- Volume-depleted patients or those who, in the judgement of the investigator, may be at risk for dehydration (abuse of diuretics or laxatives, chronic diarrhoea etc.).

- History of recurrent or serious genitor-urinary infections.

- Patients with known hypersensitivity to sitagliptin and its excipients.

- History of acute or chronic pancreatic disease.

- Patients who received any investigational new drug within the last 12 weeks.

- Severe obesity (body mass index, BMI $\geq 40 \mathrm{~kg} / \mathrm{m}^{2}$ ).

- Uncontrolled blood pressure, defined as $>$ 160/100 mmHg.

- Expected glomerular filtration rate (eGFR) $<45 \mathrm{ml} /$ $\min / 1.73 \mathrm{~m}^{2}$.
- Severe hepatic insufficiency and/or significant abnormal liver function defined as aspartate aminotransferase (AST) $>3 \times$ upper limit of normal (ULN) and/ or alanine aminotransferase (ALT) $>3 \times$ ULN or total bilirubin $>2.0 \mathrm{mg} / \mathrm{dl}$.

- Cardiac arrhythmia (2nd grade AV block, atrial fibrillation, pacemaker, high incidence premature beats).

- Clinically relevant cardiac valvular disease.

- Ejection fraction $<50 \%$ or presence of regional left ventricular contraction impairment.

- Poor acoustic window or poor quality of echocardiographic imaging.

- Inability to perform the cardiopulmonary exercise test.

- Evidence of inducible myocardial ischemia at the cardiopulmonary test.

\section{Randomisation and treatment allocation}

Patients will be randomized to a 26-week treatment with either empagliflozin $10 \mathrm{mg}$ or sitagliptin $100 \mathrm{mg} /$ daily as add-on to the background therapy. This will be done with a randomization matrix of 75 numbers calculated using the web-based service at http://www.random.org, assigning pair and even numbers to empagliflozin and sitagliptin, respectively. If the patient requires additional treatment for the presence of hyperglycemia, either symptomatic or detected at self-glucose monitoring (fasting plasma glucose $(\geq 10 \mathrm{mM})$, the following measures will be sequentially taken: (a) lifestyle measures reinforcement, (b) initiation or up titration of metformin, (c) 
initiation or up titration of basal insulin, (d) withdrawal from the trial.

\section{Study objectives \\ Primary objective}

To verify whether, in our population of T2D patients with normal 2-D EF ( $\geq 50 \%)$ and without evidence of cardiac valvular or ischemic disease, the chronic treatment with empagliflozin is associated with an improvement in LV systolic function as measured by GLS through STE in comparison to sitagliptin, an equally effective plasma glucose lowering agent presumably neutral on cardiac function.

\section{Secondary objectives}

1. To compare the effects of the 2 treatments on:

- 3D echo left ventricular systolic and diastolic functional parameters

- Plasma mechanism-oriented biomarkers

- $\mathrm{VO}_{2} \max$

- Cardiac autonomic function tests.

2. To test whether the effect of empagliflozin differs in the subgroup of patients with more pronounced abnormalities in cardiac systolic function at baseline (GLS > - 18\%).

3. To assess in the whole cohort the relationship between the changes in cardiac function indices (GLS or 3D) and the concomitant changes in cardiac biomarkers and/or in blood pressure, weight and degree of metabolic control (exploratory analysis on the mechanisms).

\section{Study endpoints}

\section{Primary outcome variable}

Changes in global longitudinal strain (GLS) from baseline to 1 and 6 months after treatment initiation.

\section{Secondary outcome variables}

Changes from baseline at 6 months after treatment initiation in

1. 3-D Echocardiography EF, left atrial volume and $E / E^{\prime}$.

2. $\mathrm{VO}_{2} \max$, as measured at cardiopulmonary test.

3. Myocardial parietal stress plasma biomarkers (BNP, NT-proBNP, proadrenomedullin), inflammation/oxidative stress plasma biomarkers (hsCRP, TNF-alpha, mieloperoxidase, uric acid) and cardiac remodelling/cytolysis biomarkers (type III pro-collagen, troponine), body volume biomarkers (total body water, plasma renin and aldosterone).
4. Cardiac autonomic function tests $(\mathrm{R}-\mathrm{R}$ interval during Valsalva manoeuvre, deep-breathing, lying-tostanding).

\section{Exploratory pre-defined hypothesis-driven analyses}

1. Whether changes from baseline at 6 months after treatment initiation in plasma markers of volume control (plasma aldosterone-to-renin ratio and total body water), cardiomyocyte strain (BNP, NTproBNP, proadrenomedullin), inflammation/oxidative stress (hsCRP, TNF- $\alpha$, myeloperoxydase, uric acid), matrix remodeling (procollagen type III) and myocyte injury (Troponin T) help understanding the mechanisms of action through which the treatments exert their effect/s on the heart.

2. Whether changes from baseline at 6 months after treatment initiation in cardiac autonomic function score (based on RR changes with Valsalva, deep breathing, standing-to-laying) contribute to the mechanisms of action of the treatments.

3. Whether the effects of the treatments differ in the subgroup of patients whom at baseline have mild abnormalities in cardiac systolic function or abnormal values of plasma biomarkers or abnormal cardiac autonomic function tests [lower 50 percentile of GLS, atrial enlargement (LAVI $\left.>34 \mathrm{ml} / \mathrm{m}^{2}\right)$, LV hypertrophy (LVMI $>115$ or $90 \mathrm{~g} / \mathrm{m} 2$, males and females, respectively), diastolic dysfunction $\left(E / \mathrm{e}^{\prime} \geq 13\right)$ and myocardial strain (Nt-pro-BNP $>125 \mathrm{pg} / \mathrm{ml})$ ].

4. Whether the changes in cardiac function are dependent on the concomitant changes in blood pressure, body weight and/or the improvement of the metabolic control and/or fasting plasma $\mathrm{B}-\mathrm{OH}$ butirrate concentration.

\section{Power calculation and sample size}

The size of a change in GLS that is considered clinical relevant is based on the following data: (a) mean GLS $( \pm$ SD) as assessed in a population of 114 type 2 diabetic subjects without evidence of cardiac disease and with $2 \mathrm{D}-\mathrm{EF} \geq 55 \%$ according to (15) was: $-19( \pm 3) \%$; (b) a difference in GLS of $2.5 \%(-21.2 \pm 2.7$ vs $-18.7 \pm 2.8 \%)$ was observed between stage A and stage B heart failure in non-diabetic patients [20], and (c) a similar difference is present between subjects with and without diabetes [48]. A difference $\geq 2.5 \%$ is therefore considered highly clinically meaningful. Given the nature of the study (within subject repeated measures) a difference $\geq 1.5 \%$ would be considered relevant from a pathophysiologic point of view. Alpha error is always kept at 0.05. Estimation of the SD of the difference is based on an observational 
study performed in 112 type 2 diabetic patients with normal EF in whom after 2 years the GLS changed from $-19.7 \pm 4.0$ to $-18.9 \pm 3.8 \%$ a difference of $0.8 \%$, which was statistically significant $(\mathrm{p}<0.001)$ at a paired $\mathrm{t}$-test. The SD of the difference in GLS is not reported, however it can be estimated using a web-based engine (http:// www.biomath.info/power/prt.htm) on the bases of the available mean and SD and by attributing a value to the correlation between the two repeated measurements of 0.80 on the bases of the intra observed error in GLS measurement $0.7 \pm 0.4 \%$ according to Kosmala et al. [20] and on personal data. This data allowed the SD to be estimated as $2.5 \%$.

Therefore, to detect an absolute $\geq 2.5 \%$ difference in GLS, a sample size of 30 subjects is required per arm. Considering a $21 \%$ drop-out, sample size will be 75 recruited subjects, to have 60 completed. In terms of Power for a total sample size of 60 (2 groups of 30), given the above-mentioned figures in a paired design (either t-test or Wilcoxon) within each of the two treatment groups $(\mathrm{n}=30)$ the power to detect a difference of 2.5 and $1.7 \%$ is 0.99 and 0.95 , respectively. In an
MANOVA design for three repeated measures (baseline, 1, 6 months) the power of the study to detect a difference between the treatments of 2.5 or $1.7 \%$ is 0.98 and 0.80 , respectively. In an ANCOVA design with 4 covariates a sample size of 60 subjects will have the power of 0.71 to detect an effect of 0.10 (expressed as of proportion of variance explained by the effect under consideration), which is commonly considered small in biostatistics and a power of 0.99 to detect a medium effect $(0.25)$. The analysis will be 2 -sided, PPS, no interim.

\section{Discussion}

With this study we aim at demonstrating whether the treatment with empagliflozin is associated with the reversal of the earliest signs of diabetic systolic and diastolic dysfunctions, and at shedding light on the underlying mechanism/s involved (Fig. 2).

The reasons for concentrating on the earliest stages of HF stems from the common idea that this condition is more susceptible to reversal and also from the data of the EMPAREG OUTCOME trial showing the more robust effects, both in terms of hospitalization for HF

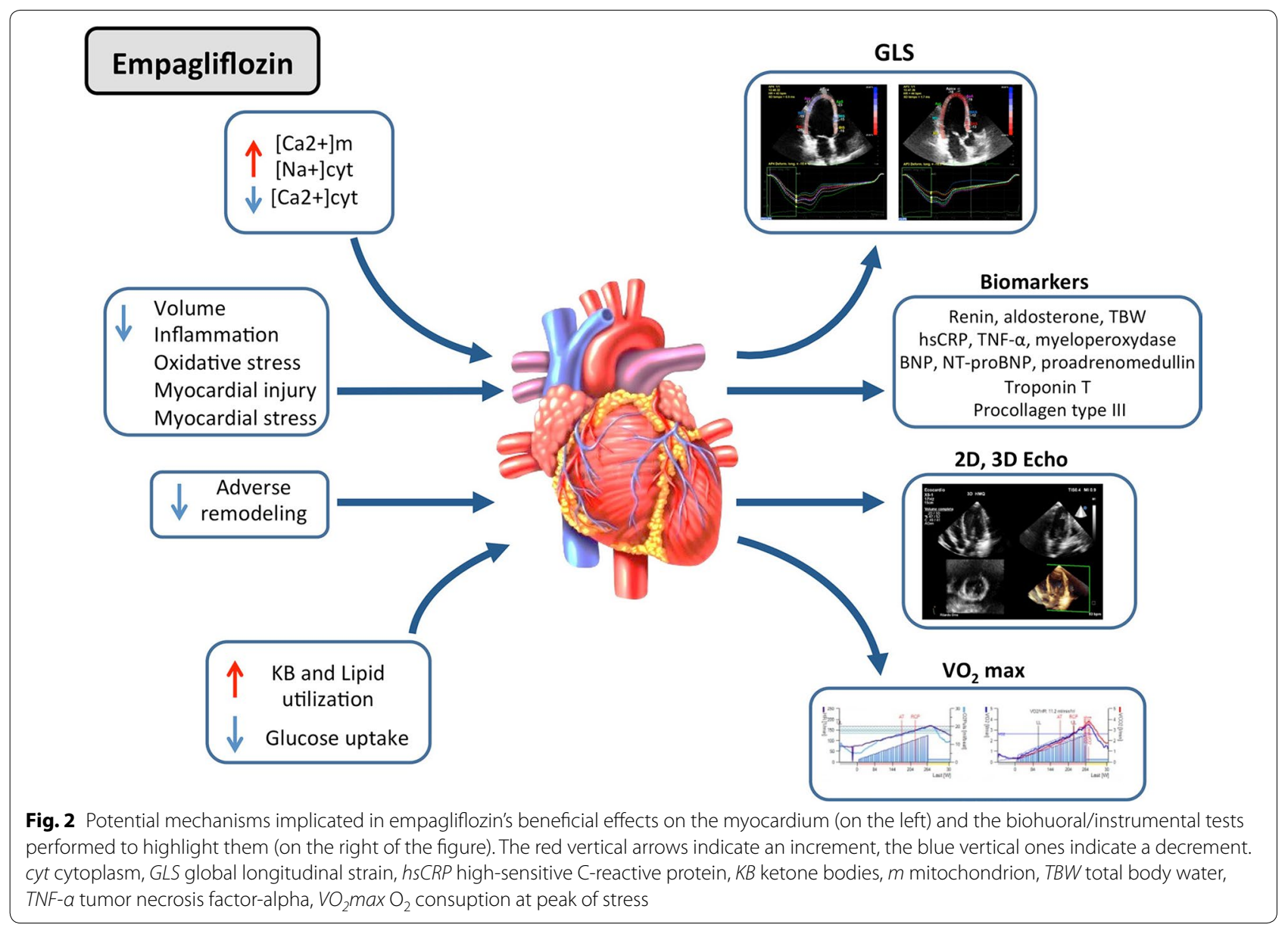


and cardiovascular death, in the patients without HF at baseline or diagnosed during the study [10]. We therefore focused on patients with normal EF without current or prior symptoms of overt HF, but at high risk for the condition for presence of T2D and allowing for arterial hypertension and structural (LV hypertrophy) or functional (diastolic dysfunction) or biochemical (NT-pro$\mathrm{BNP}$ ) evidence of initial myocardial disease (Stage A and $\mathrm{B}$ HF according to ACC/AHA guidelines). In terms of pathophysiological changes the data are essentially based on few experimental studies and speculations [49]. SGLT2 inhibitors might actually affect myocardial functions through mechanisms that can act both directly and indirectly on the myocardium. Chronic treatment with SGLT2 inhibitors has been associated to a small blood pressure reduction $(3-4 \mathrm{mmHg}$ mean blood pressure) also confirmed by 24-h blood pressure monitoring [50], which was not associated to an increase in heart rate [51] and was independent of the number and type of baseline anti-hypertensive drugs [52]. The reasons for this blood pressure reduction, though not fully understood, is likely to involve the small (5\%) weight reduction and the minor diuretic and natriuretic effects $[50,51]$ that are induced by glycosuria. Given the size of these changes, however, it is unlikely that they entirely justify the clinical outcomes. Since SGLT2 inhibitors reduce arterial blood pressure without a compensatory increase in heart rate, a reduction in the sympathetic tone [53] has also been suggested as a potential mechanism. Owing to its renal and hemodynamic effects, also other neurohormonal axes might be involved, particularly the RAAS and NPs, resulting in a change in TBW. To date, however, at the best of our knowledge no data is available. With regard to body fluid control the information is limited to a single preliminary study in which chronic treatment with dapagliflozin resulted in a $5 \%$ reduction in plasma volume [50]. A direct vascular effect might also contribute to blood pressure changes. Interestingly, a 8-week empagliflozin treatment reduced arterial stiffness in patients with type 1 diabetes [54]. Preliminary experimental data suggest that SGLT2 inhibitors may improve the vascular structural properties, interfering with collagen, elastin, advanced glycation end-products [55]. Moreover, the SGLT2 inhibitors-induced increased ketone bodies has been proposed to facilitate myocardial energetics [56]. In fact, myocardial utilization of $\beta-\mathrm{OH}$-butirrate results in a significant increase in ATP production with respect to glucose and fatty acid oxidation and in an improved efficiency (hydraulic work/energy from $\mathrm{O}_{2}$ consumed) in a model of isolated working heart by $25 \%$ [57].

Together with the above-mentioned indirect effects, empagliflozin shows some direct myocardial effects as well. In cardiomyocytes, mitochondrial $\mathrm{Ca}^{2+}$ is considered to be one major activator of ATP synthesis and of the antioxidant enzymatic network [58]. Elevated cardiac cytoplasmic $\mathrm{Na}^{+}$and $\mathrm{Ca}^{2+}$ concentrations and decreased mitochondrial $\mathrm{Ca}^{2+}$ concentration are characteristic hyperglycemia-induced drivers of $\mathrm{HF}$ and cardiac death. In a recent paper [59] empagliflozin was demonstrated to reduce cardiomyocytes cardiac cytoplasmic $\mathrm{Na}^{+}$and $\mathrm{Ca}^{2+}$ concentrations and to increase mitochondrial $\mathrm{Ca}^{2+}$ levels by directly inhibiting $\mathrm{Na}^{+} / \mathrm{H}^{+}$exchanger (NHE). Previous studies have demonstrated that chronic inhibition of NHE prevents or mitigates HF in animal models [60], and this might be one of the mechanisms through which empagliflozin protects from HF deaths and hospitalizations. Finally, recent observation raised the possibility that SGLT2 inhibitor treatment might also have a beneficial effect on cardiac remodeling. In a rat model of progressive HF, it has been observed that SGLT2 inhibition could attenuate the increase in LV mass and $\mathrm{LV}$ end-diastolic diameter [61]. In obese T2D $(\mathrm{db} / \mathrm{db})$ mice, a 10 week-long treatment with empagliflozin has been observed to reduce interstitial cardiac fibrosis, pericoronary arterial fibrosis, coronary arterial thickening, cardiac interstitial macrophage infiltration, and cardiac superoxide levels [62]. In the same study, empagliflozin also ameliorated endothelial dysfunction. Moreover, in animal models, SGLT-2 inhibitors have been shown to reduce myocardial leukocytosis induced by hyperglycemia [63] and to reduce inflammation and oxidative stress [64] as well as possibly fibrosis [65]. The only study that is available on cardiac function is a preliminary observation done in 10 T2D patients by using standard 2D echocardiography, that reported a small improvement in LV mass (reverse remodeling) and diastolic function after 5 months of treatment with empagliflozin [21]. To date, no data on the effect of SGLT2 inhibitors on cardiac remodeling in subjects with HF is available, but the REFORM trial [66] is currently underway in order to investigate, in patients with T2D and HF, the effects of dapagliflozin on LV remodeling through cardiac magnetic resonance and cardiopulmonary stress test.

\section{Limitations}

Despite the estimate high power of the study, this is an open, short-term, and relatively small trial. In order to prevent any influence of the researcher expectations (type 1 error) we will take care that the cardiologist performing the primary and several of the secondary outcome variables measurements are blind with respect to the treatment allocation. In addition, given the expected mild degree of systolic dysfunction at baseline caused by our strict selection criteria we are somehow protected from type 1 error although the risk to occur in a type 2 error is increased. However, by measuring a full set of 
other relevant myocardial function parameters and biomarkers we will actually be able to generate solid datadriven hypotheses for future studies. The recent evidence of the PROLOGUE trial (Yamada), showing that sitagliptin is able to prevent the time-related deterioration of diastolic function, raises the possibility that our comparator is not completely neutral on cardiac function. However, this effect was evident after 12, but not after 6 months of treatment and sitagliptin has been shown to be neutral on systolic function in patients with postischemic heart failure with mildly reduced ejection fraction [67]. Therefore, there will be, if any, a small risk of type 2 error, which we consider acceptable and eventually would, in case, reinforce any observed positive outcome of empagliflozin.

\section{Abbreviations}

BMI: body mass index; BNP: brain-derived natriuretic peptide; EF: ejection fraction; eGFR: expected glomerular filtration rate; GLS: global longitudinal strain; HF: heart failure; hsCRP: high-sensitive C-reactive protein; LV: left ventricle; MPO: myeloperoxidase; NHE: $\mathrm{Na}^{+} / \mathrm{H}^{+}$exchanger; NPs: natriuretic peptides; NT-proBNP: N-terminal-proBNP; RAAS: renin-angiotensin-aldosterone system; SGLT2: sodium-glucose cotransporter 2; STE: speckle-tracking echocardiography; T2D: type 2 diabetes; TBW: total body water; TNF-a: tumor necrosis factor-alpha; $\mathrm{VO}_{2}$ max: maximal oxygen volume.
\end{abstract}

\section{Authors' contributions}

AN: Study design, manuscript revision. LN: Manuscript preparation. IF, EC: Echocardiography and cardio pulmonary test description. VDB: Manuscript revision, statistics. All authors read and approved the final manuscript.

\section{Author details}

1 Department of Clinical and Experimental Medicine, Pisa University, Via Savi 27, 56100 Pisa, Italy. ${ }^{2}$ Department of Surgery, Medical, Molecular, and Critical Area Pathology, Pisa University, Pisa, Italy.

\section{Acknowledgements}

None.

\section{Competing interests}

The authors declare that they have no competing interests.

\section{Availability of data and materials}

Data sharing is not applicable to this article as no datasets were generated or analysed during the current study.

\section{Consent for publication}

The paper contains no human data, so there is no need for consent for publication.

\section{Ethics approval and consent to participate}

Application to the National Regulatory Authorities for approval of this study is made according to specific National regulations (DL 51 of 21/12/2007). A copy of the approval will be archived in the study master file in the local study file of the Investigator. Furthermore, the study has been submitted to the Hospital/ University Ethical Committee ("Comitato Etico Area Vasta Nord-Ovest" at the Pisa University Hospital (AOUP), Via Roma 67, 56126 Pisa, Italy) for approval. Financial agreement will be reached with Hospital Administration before initiation of the trial. The study will be conducted under the provision of the "D.M. 17.12.2004" for the conduction of no-profit studies and according to the DL 211 of 24/06/2003.

The study will be conducted in accordance with the Declaration of Helsinki (1964) and its amendments. In seeking informed consent, the Investigator will inform the subject that participation to the trial is voluntary and that refusal will not lead to loss of any benefit or prejudice the relationship with the physician in any way. Furthermore, a statement will be made to the effect that withdrawal from the trial is possible at any moment without having to give a specific reason. Before enrolment into the trial, each subject will receive a full explanation of the nature and purpose of the study from the Investigator, together with a description of benefits and risks associated with participation; insurance coverage will also be mentioned and related procedures in the event of injury will be explained. A clear Information Sheet covering all important aspects in writing will be given to the subject who will read it and has the opportunity to ask any questions. The subject will be given adequate time for consideration before he/she is requested to sign the consent form in duplicate. One of the original copies of the signed consent form will be kept by the Investigator in the study file. The subject will receive the other one for future reference.

\section{Funding}

The study is an investigator-initiated study supported at $50 \%$ by an unrestricted grant from Boehringer Ingelheim.

\section{Publisher's Note}

Springer Nature remains neutral with regard to jurisdictional claims in published maps and institutional affiliations.

Received: 22 July 2017 Accepted: 5 October 2017

Published online: 12 October 2017

\section{References}

1. Haider AW, Larson MG, Franklin SS, Levy D. Systolic blood pressure, diastolic blood pressure, and pulse pressure as predictors of risk for congestive heart failure in the Framingham Heart Study. Ann Intern Med. 2003;138:10-6

2. Goyal BR, Mehta AA. Diabetic cardiomyopathy: pathophysiological mechanisms and cardiac dysfuntion. Hum Exp Toxicol. 2013;32:571-90.

3. Piepoli MF, Hoes AW, Agewall S, et al. 2016 European Guidelines on cardiovascular disease prevention in clinical practice: the Sixth Joint Task Force of the European Society of Cardiology and Other Societies on Cardiovascular Disease Prevention in Clinical Practice (constituted by representatives of 10 societies and by invited experts)Developed with the special contribution of the European Association for Cardiovascular Prevention \& Rehabilitation (EACPR). Eur Heart J. 2016;37:2315-81.

4. Faden G, Faganello G, De Feo S, et al. The increasing detection of asymptomatic left ventricular dysfunction in patients with type 2 diabetes mellitus without overt cardiac disease: data from the SHORTWAVE study. Diabetes Res Clin Pract. 2013;101:309-16.

5. Cioffi G, Giorda CB, Chinali M, et al. Analysis of midwall shortening reveals high prevalence of left ventricular myocardial dysfunction in patients with diabetes mellitus: the DYDA study. Eur J Prev Cardiol. 2012;19:935-43.

6. Rahmoune H, Thompson PW, Ward JM, Smith CD, Hong G, Brown J. Glucose transporters in human renal proximal tubular cells isolated from the urine of patients with non-insulin-dependent diabetes. Diabetes. 2005;54:3427-34.

7. Solini A, Rossi C, Mazzanti CM, Proietti A, Koepsell H, Ferrannini E. SGLT2 and SGLT1 renal expression in patients with type 2 diabetes. Diabetes Obes Metab. 2017:19(9):1289-94.

8. Zhou J, Xu J, Huang Z, Wang M. Transporter-mediated tissue targeting of therapeutic molecules in drug discovery. Bioorg Med Chem Lett. 2015;25:993-7.

9. Schwartz SS, Ahmed I. Sodium-glucose cotransporter 2 inhibitors: an evidence-based practice approach to their use in the natural history of type 2 diabetes. Curr Med Res Opin. 2016;32:907-19.

10. Zinman B, Wanner C, Lachin JM, et al. Empagliflozin, Cardiovascular Outcomes, and Mortality in Type 2 Diabetes. N Engl J Med. 2015;373:2117-28.

11. Dormandy JA, Charbonnel B, Eckland DJ, et al. Secondary prevention of macrovascular events in patients with type 2 diabetes in the PROactive Study (PROspective pioglitAzone Clinical Trial In macroVascular Events): a randomised controlled trial. Lancet. 2005;366:1279-89. 
12. Cavender MA, Scirica BM, Raz I, et al. Cardiovascular outcomes of patients in SAVOR-TIMI 53 by baseline hemoglobin A1c. Am J Med. 2016;129(340):e341-8

13. Green JB, Bethel MA, Armstrong PW, et al. Effect of sitagliptin on cardiovascular outcomes in type 2 diabetes. N Engl J Med. 2015;373:232-42.

14. Hirakawa Y, Arima H, Zoungas S, et al. Impact of visit-to-visit glycemic variability on the risks of macrovascular and microvascular events and all-cause mortality in type 2 diabetes: the ADVANCE trial. Diabetes Care. 2014;37:2359-65.

15. Neal B, Perkovic V, Mahaffey KW, et al. Canagliflozin and cardiovascular and renal events in type 2 diabetes. N Engl J Med. 2017:377:644-57.

16. Kosiborod M, Cavender MA, Fu AZ, et al. Lower Risk of Heart Failure and Death in Patients Initiated on Sodium-Glucose Cotransporter-2 Inhibitors Versus Other Glucose-Lowering Drugs: the CVD-REAL Study (Comparative Effectiveness of Cardiovascular Outcomes in New Users of SodiumGlucose Cotransporter-2 Inhibitors). Circulation. 2017;136:249-59.

17. Januzzi JL Jr, Butler J, Jarolim P, et al. Effects of canagliflozin on cardiovascular biomarkers in older adults with type 2 diabetes. J Am Coll Cardiol. 2017;70:704-12.

18. Onishi T, Saha SK, Delgado-Montero A, et al. Global longitudinal strain and global circumferential strain by speckle-tracking echocardiography and feature-tracking cardiac magnetic resonance imaging: comparison with left ventricular ejection fraction. J Am Soc Echocardiogr. 2015;28:587-96.

19. Marwick TH, Leano RL, Brown J, et al. Myocardial strain measurement with 2-dimensional speckle-tracking echocardiography: definition of normal range. JACC Cardiovasc Imaging. 2009;2:80-4.

20. Kosmala W, Jellis CL, Marwick TH. Exercise limitation associated with asymptomatic left ventricular impairment: analogy with stage B heart failure. J Am Coll Cardiol. 2015;65:257-66.

21. Verma S, Garg A, Yan AT, et al. Effect of empagliflozin on left ventricular mass and diastolic function in individuals with diabetes: an important clue to the EMPA-REG OUTCOME trial? Diabetes Care. 2016:39:e212-3.

22. Abduch MCD, Alencar AM, Mathias W, Vieira MLD. Cardiac mechanics evaluated by speckle tracking echocardiography. Arq Bras Cardiol. 2014;102:403-12.

23. Zhang $X$, Wei X, Liang Y, Liu M, Li C, Tang H. Differential changes of left ventricular myocardial deformation in diabetic patients with controlled and uncontrolled blood glucose: a three-dimensional speckletracking echocardiography-based study. J Am Soc Echocardiogr. 2013;26:499-506

24. Ng AC, Sitges M, Pham PN, et al. Incremental value of 2-dimensional speckle tracking strain imaging to wall motion analysis for detection of coronary artery disease in patients undergoing dobutamine stress echocardiography. Am Heart J. 2009;158:836-44.

25. Enomoto $M$, Ishizu T, Seo $Y$, et al. Subendocardial systolic dysfunction in asymptomatic normotensive diabetic patients. Circ J. 2015;79:1749-55.

26. Holland DJ, Marwick TH, Haluska BA, et al. Subclinical LV dysfunction and 10-year outcomes in type 2 diabetes mellitus. Heart. 2015;101:1061-6.

27. Maeder MT, Karapanagiotidis S, Dewar EM, Kaye DM. Accuracy of echocardiographic cardiac index assessment in subjects with preserved left ventricular ejection fraction. Echocardiography. 2015;32:1628-38.

28. Braunwald E. Biomarkers in heart failure. N Engl J Med. 2008;358:2148-59.

29. Vergaro $G$, Emdin M, lervasi $A$, et al. Prognostic value of plasma renin activity in heart failure. Am J Cardiol. 2011;108:246-51.

30. Lichtenbelt WDV, Westerterp KR, Wouters L. Deuterium dilution as a method for determining total-body water-effect of test protocol and sampling time. Brit J Nutr. 1994;72:491-7.

31. Schoeller DA, van Santen E, Peterson DW, Dietz W, Jaspan J, Klein PD. Total body water measurement in humans with $18 \mathrm{O}$ and $2 \mathrm{H}$ labeled water. Am J Clin Nutr. 1980;33:2686-93.

32. Khaled MA, Lukaski HC, Watkins CL. Determination of total body water by deuterium NMR. Am J Clin Nutr. 1987;45:1-6.

33. Daniels LB, Maisel AS. Natriuretic peptides. J Am Coll Cardiol. 2007;50:2357-68.

34. Vickery S, Price CP, John Rl, et al. B-type natriuretic peptide (BNP) and amino-terminal proBNP in patients with CKD: relationship to renal function and left ventricular hypertrophy. Am J Kidney Dis. 2005;46:610-20.

35. Nagaya N, Satoh T, Nishikimi T, et al. Hemodynamic, renal, and hormonal effects of adrenomedullin infusion in patients with congestive heart failure. Circulation. 2000;101:498-503.
36. Jougasaki M, Wei CM, McKinley LJ, Burnett JC Jr. Elevation of circulating and ventricular adrenomedullin in human congestive heart failure. Circulation. 1995;92:286-9.

37. Sugiura T, Takase H, Toriyama T, Goto T, Ueda R, Dohi Y. Circulating levels of myocardial proteins predict future deterioration of congestive heart failure. J Card Fail. 2005;11:504-9.

38. Castell JV, Gomez-Lechon MJ, David M, Fabra R, Trullenque R, Heinrich PC. Acute-phase response of human hepatocytes: regulation of acute-phase protein synthesis by interleukin-6. Hepatology. 1990;12:1179-86.

39. Anand IS, Latini R, Florea VG, et al. C-reactive protein in heart failure: prognostic value and the effect of valsartan. Circulation. 2005;112:1428-34.

40. Vasan RS, Sullivan LM, Roubenoff R, et al. Inflammatory markers and risk of heart failure in elderly subjects without prior myocardial infarctionthe Framingham Heart Study. Circulation. 2003;107:1486-91.

41. Venugopal SK, Devaraj S, Jialal I. Effect of C-reactive protein on vascular cells: evidence for a proinflammatory, proatherogenic role. Curr Opin Nephrol Hypertens. 2005;14:33-7.

42. Anker SD. Inflammatory mediators in chronic heart failure: an overview. Heart. 2004;90:464-70.

43. Mann DL, McMurray JJ, Packer M, et al. Targeted anticytokine therapy in patients with chronic heart failure: results of the Randomized Etanercept Worldwide Evaluation (RENEWAL). Circulation. 2004;109:1594-602.

44. Lee DS, Vasan RS. Novel markers for heart failure diagnosis and prognosis. Curr Opin Cardiol. 2005:20:201-10.

45. Kameda K, Matsunaga T, Abe N, et al. Correlation of oxidative stress with activity of matrix metalloproteinase in patients with coronary artery disease. Possible role for left ventricular remodelling. Eur Heart J. 2003;24:2180-5.

46. Tang WH, Brennan ML, Philip K, et al. Plasma myeloperoxidase levels in patients with chronic heart failure. Am J Cardiol. 2006;98:796-9.

47. Cicoira M, Rossi A, Bonapace S, et al. Independent and additional prognostic value of aminoterminal propeptide of type III procollagen circulating levels in patients with chronic heart failure. J Card Fail. 2004;10:403-11.

48. Ernande L, Bergerot C, Rietzschel ER, et al. Diastolic dysfunction in patients with type 2 diabetes mellitus: is it really the first marker of diabetic cardiomyopathy? J Am Soc Echocardiogr. 2011:24(1268-1275):e1261.

49. Heerspink HJ, Perkins BA, Fitchett DH, Husain M, Cherney DZ. sodium glucose cotransporter 2 inhibitors in the treatment of diabetes mellitus: cardiovascular and kidney effects, potential mechanisms, and clinical applications. Circulation. 2016;134:752-72

50. Lambers Heerspink HJ, de Zeeuw D, Wie L, Leslie B, List J. Dapagliflozin a glucose-regulating drug with diuretic properties in subjects with type 2 diabetes. Diabetes Obes Metab. 2013;15:853-62.

51. Vasilakou D, Karagiannis T, Athanasiadou E, et al. Sodium-glucose cotransporter 2 inhibitors for type 2 diabetes: a systematic review and meta-analysis. Ann Intern Med. 2013;159:262-74.

52. Zoungas S, Chalmers J, Neal B, et al. Follow-up of blood-pressure lowering and glucose control in type 2 diabetes. N Engl J Med. 2014:371:1392-406.

53. Schlaich M, Elliott R, Rudnicka C, Matthews V (2016) Os 33-04 regulation of Sglt-2 by the sympathetic nervous system. J Hypertens 34 Suppl 1 ISH 2016 Abstract Book: e394.

54. Cherney DZ, Perkins BA, Soleymanlou N, et al. The effect of empagliflozin on arterial stiffness and heart rate variability in subjects with uncomplicated type 1 diabetes mellitus. Cardiovasc Diabetol. 2014;13:28.

55. Zimlichman R. Treatment of hypertension and metabolic syndrome: lowering blood pressure is not enough for organ protection, new approacharterial destiffening. Curr Hypertens Rep. 2014;16:479.

56. Mudaliar S, Alloju S, Henry RR. Can a shift in fuel energetics explain the beneficial cardiorenal outcomes in the EMPA-REG outcome study? A unifying hypothesis. Diabetes Care. 2016;39:1115-22.

57. Sato K, Kashiwaya Y, Keon CA, et al. Insulin, ketone bodies, and mitochondrial energy transduction. FASEB J. 1995;9:651-8.

58. Kohlhaas M, Liu T, Knopp A, et al. Elevated cytosolic Na+ increases mitochondrial formation of reactive oxygen species in failing cardiac myocytes. Circulation. 2010;121:1606-13.

59. Baartscheer A, Schumacher CA, Wust RC, et al. Empagliflozin decreases myocardial cytoplasmic $\mathrm{Na}+$ through inhibition of the cardiac $\mathrm{Na}+/ \mathrm{H}+$ exchanger in rats and rabbits. Diabetologia. 2017;60:568-73. 
60. Baartscheer A, Hardziyenka M, Schumacher CA, et al. Chronic inhibition of the $\mathrm{Na}(+) / \mathrm{H}(+)$-exchanger causes regression of hypertrophy, heart failure, and ionic and electrophysiological remodelling. Brit J Pharmacol. 2008;154:1266-75.

61. Abbasi F, Chu JW, McLaughlin T, Lamendola C, Leary ET, Reaven GM. Effect of metformin treatment on multiple cardiovascular disease risk factors in patients with type 2 diabetes mellitus. Metab Clin Exp. 2004;53:159-64.

62. Lin B, Koibuchi N, Hasegawa $Y$, et al. Glycemic control with empagliflozin, a novel selective SGLT2 inhibitor, ameliorates cardiovascular injury and cognitive dysfunction in obese and type 2 diabetic mice. Cardiovasc Diabetol. 2014;13:148.

63. Nagareddy PR, Murphy AJ, Stirzaker RA, et al. Hyperglycemia promotes myelopoiesis and impairs the resolution of atherosclerosis. Cell Metab. 2013;17:695-708.

64. Tahara A, Kurosaki E, Yokono M, et al. Effects of sodium-glucose cotransporter 2 selective inhibitor ipragliflozin on hyperglycaemia, oxidative stress, inflammation and liver injury in streptozotocin-induced type 1 diabetic rats. J Pharm Pharmacol. 2014;66:975-87.

65. Gallo LA, Ward MS, Fotheringham AK, et al. Once daily administration of the SGLT2 inhibitor, empagliflozin, attenuates markers of renal fibrosis without improving albuminuria in diabetic $\mathrm{db} / \mathrm{db}$ mice. Sci Rep. 2016;6:26428.

66. Singh JS, Fathi A, Vickneson K, et al. Research into the effect Of SGLT2 inhibition on left ventricular remodelling in patients with heart failure and diabetes mellitus (REFORM) trial rationale and design. Cardiovasc Diabetol. 2016;15:97.

67. Yamada H, Tanaka A, Kusunose K, et al. Effect of sitagliptin on the echocardiographic parameters of left ventricular diastolic function in patients with type 2 diabetes: a subgroup analysis of the PROLOGUE study. Cardiovasc Diabetol. 2017;16:63.

\section{Submit your next manuscript to BioMed Central and we will help you at every step:}

- We accept pre-submission inquiries

- Our selector tool helps you to find the most relevant journal

- We provide round the clock customer support

- Convenient online submission

- Thorough peer review

- Inclusion in PubMed and all major indexing services

- Maximum visibility for your research

Submit your manuscript at www.biomedcentral.com/submit 